\title{
Gravity Drainage Visualization Experimental Study of Heavy Oil Reservoirs
}

\author{
Lili Liu, Shuren Yang, Chunsheng Wang, Qiji Sun, Xiaojun Zhong \\ College of Petroleum Engineering, Northeast Petroleum University, Daqing, China \\ Email: dqliull2009@163.com
}

Received 13 April 2016; accepted 8 May 2016; published 11 May 2016

Copyright (C) 2016 by authors and Scientific Research Publishing Inc.

This work is licensed under the Creative Commons Attribution International License (CC BY). http://creativecommons.org/licenses/by/4.0/

(c) (i) Open Access

\begin{abstract}
Liaohe oilfield gravity drainage assisted steam flooding of heavy oil reservoir has made significant development effect, but the drain rule of condensate is unclear in the process of development. Heavy oil drainage microscopic visualization experimental study of using core model of glass etching, drainage process simulation of heavy oil reservoir and its influence factors were analyzed. Its method turns the drainage process of images into computer numerical signal through the image acquisition system, intuitive display flow pattern of drainage, and analyses the influence of homogeneity and the pressure differential regulation of drainage through the experimental data. The experiment results show that condensate around the steam chamber has a corresponding drainage channel, not uniform or diarrhea in the gravity drainage assisted steam flooding process. At the beginning of the drainage channels formation, the instantaneous drainage amount along with the change of pressure difference is not obvious. Instantaneous drainage amount increases with increasing pressure difference in the medium term. It tends to be stable in the later. The time of drainage channels to form homogeneous core is earlier than heterogeneous core. After the drainage channel, differential effects on heterogeneous core than homogeneous core of instantaneous drainage water.
\end{abstract}

\section{Keywords}

Heavy Oil, Gravity Drainage, Visualization, Drainage Rule

\section{Introduction}

Gravity drainage technology is put forward for the first time by Liaohe oilfield, and foreign related research is less. Most of the way is by studying the mechanism of gravity drainage through laboratory experiment in the domestic. Usually used microscopic model mainly include clip particle model, rock chips and micro lithography porous media model. In the experiment process, the model is simple and feasible, and can simulate real pore 
structure. It plays an important role in the study of micro gravity drainage mechanism. However, the drawback is that gravity drainage process and effect cannot be directly observed [1]-[3]. Simulation experiments use glass etching of homogeneous and heterogeneous visual core [4]-[6]; it forms the level of reservoir model consist of pore and throat in the middle of two pieces of glass. Collecting the displacement process image by image acquisition system and translating it to computer numerical signal acquisition to study the condensate drainage rule, and the influence of pressure difference to instantaneous water amount through experimental data are analysed. The model can not only simulate the really actual reservoir gravity drainage process, but also intuitive observe the process and regularity of drainage and have a guiding significance for gravity drainage assisted steam flooding in the field.

\section{Experimentation Research}

\subsection{Experimental Purpose}

Simulating the oil-water flow regulation and characteristic analysis of the differential pressure, the influence of heterogeneity of core of discharge flow pattern of heavy oil reservoir discharge process.

\subsection{Experimental Conditions}

\subsubsection{Experimental Core}

The core model is a transparent and miniature model of glass etching ( $400 \mathrm{~mm} \times 400 \mathrm{~mm})$, using the silicone oil to change its wettability. The core model is divided into two types: homogeneous and heterogeneous, as shown in Figure 1 below.

\subsubsection{Experimental Oil}

Because of the heavy oil in the 59 block, it is difficult to oil sample saturation at the experimental temperature. Therefore, the experiment was used to get oil samples from a heavy oil block in Liaohe oil field, and the viscosity of the simulated oil at 45 centigrade is similar to the extra heavy oil at 100 centigrade, using HAAKE-RS6000 rheometer and measuring the viscosity. Curve of viscosity temperature determined by rheometer as shown in Figure 2.

\subsubsection{Experimental Temperature}

Saturated oil temperature $80^{\circ} \mathrm{C}$, Experimental temperature is $45^{\circ} \mathrm{C}$.

\subsubsection{Major Laboratory Instruments and Equipment}

The device of visual microscopic displacement experiment includes glass core, micro pump, micro visual analysis

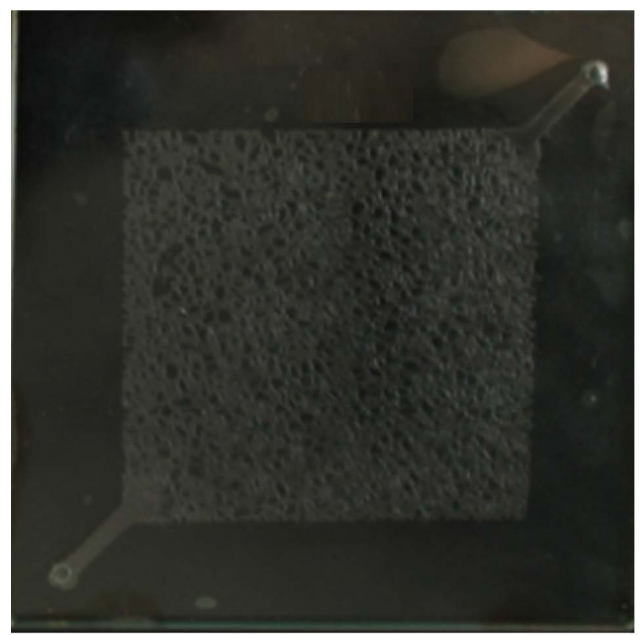

(a) Homogeneous core

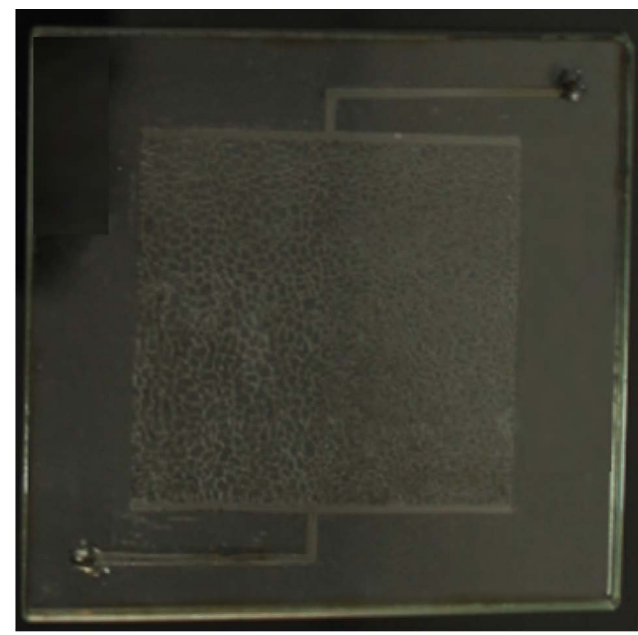

(b) Heterogeneous cores

Figure 1. Visual core model. 


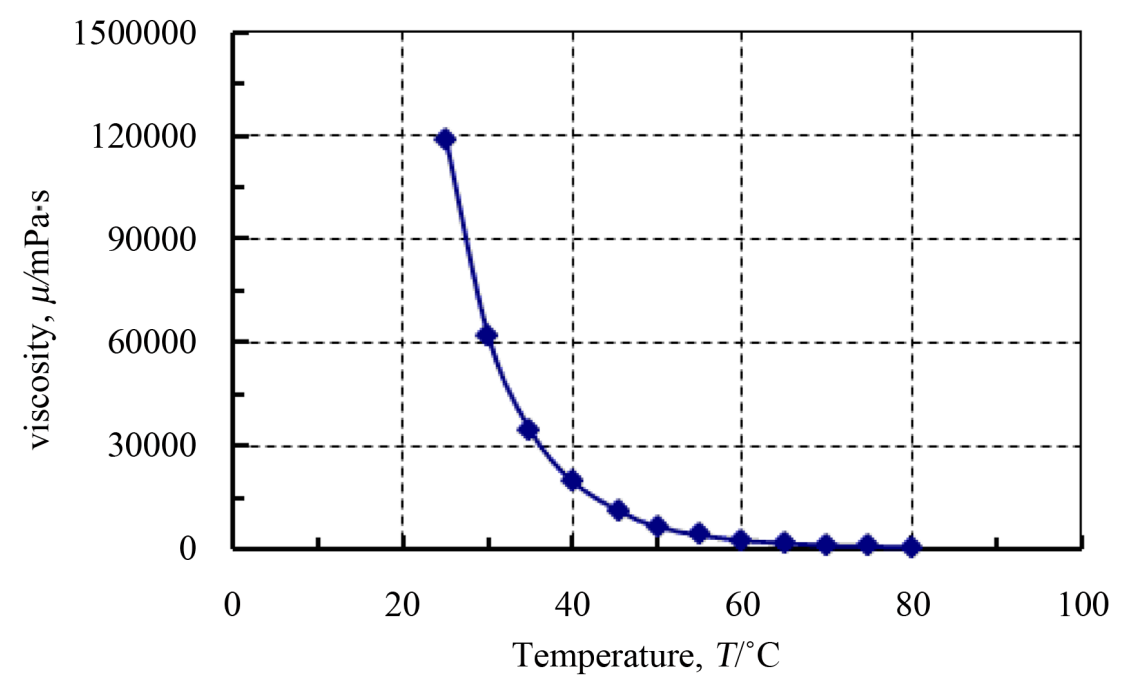

Figure 2. The viscosity-temperature curve of visual experiment.

system, image acquisition system and image analysis system.

\subsection{Experimental Procedure}

Experiments are conducted in the microscopic model incubator, through the image acquisition system to observe the flow law of oil and water under different pressure, the experiment device is shown in Figure 3.

The specific steps for the experiment:

1) Application of micro pump water saturation to microscopic model;

2) Application of trace pump will be heated to $80^{\circ} \mathrm{C}$ within the field of oil saturation to microscopic model;

3) Under the condition of temperature $45^{\circ} \mathrm{C}$, respectively, the $100 \mathrm{kPa}, 120 \mathrm{kPa}$, and $140 \mathrm{kPa}$ pressure oil displacement experiments, recording the liquid amount at all time points until the outlet end has no oil to flow out;

4) The oil water distribution and the law of oil and water flow were observed in the core model by microscope. Video displacement in the process of dynamic images, recording the image of before and after drive the displacement, divided into different core area and the remaining oil distribution of partition, using image postprocessing software for image analysis;

5) The results of the experimental data are analyzed;

6) Cleaning the core;

7) The experiment was carried out with different pressures, and the first five steps were repeated.

\subsection{Experimental Program}

The design is as follows:

1) Homogeneous core: Injection pressure is set to $100,120,140 \mathrm{kPa}$, once every 1 minute record drainage data, real-time acquisition oil-water distribution, analysis the relationship between the instantaneous water amount and time;

2) Heterogeneous cores: Injection pressure is set to 100, 120, $140 \mathrm{kpa}$, once every 2 minutes record drain data, real-time acquisition oil-water distribution, analysis the relationship between the instantaneous water amount and time.

\section{Results Analysis}

Heavy oil reservoir drainage flow visualization model of research method is mainly rely on visual direct observations and data analysis, combined with the displacement observed phenomena, in the process of residual oil formation and distribution of a qualitative description. Through the comparative analysis of flooding, flooding image, qualitative interpretation of gravity law and amount characteristics. 


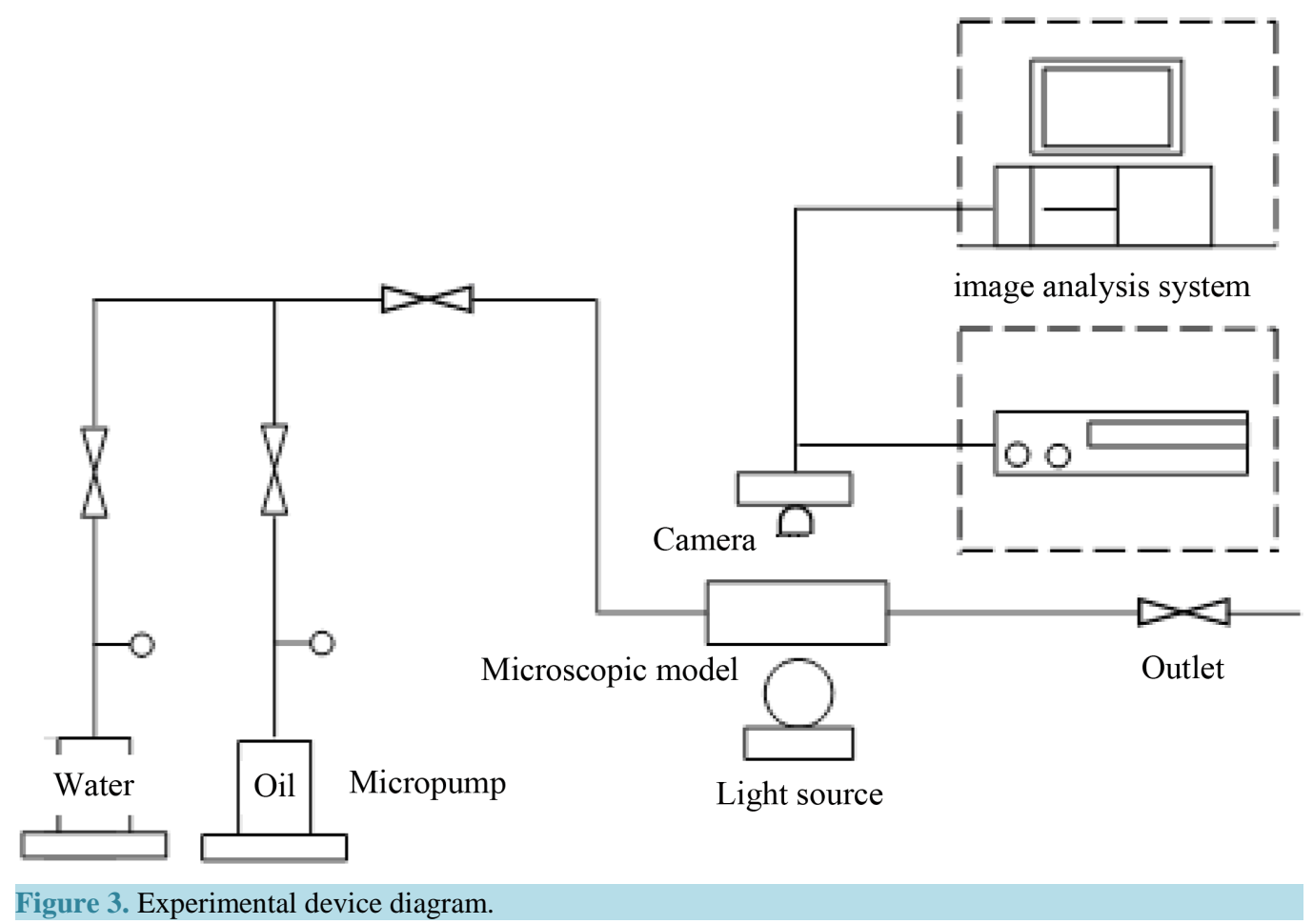

\subsection{Microscopic Visual Description of Each Period}

According to the real-time collection of pictures, choose the representative of the original state, at the beginning of the experiment, in the middle and end of the experiment the oil-water distribution map, used to describe the characteristics of amount. As shown in Figure 4 and Figure 5.

1) Homogenous model

In complicated pore structure of porous media, formed in the process of many micro flow channel, each channel of oil-water interface in different speed forward. After a period of time, only some continuous channel formed in the pore water, and quite a number of pores remain small oil region. Continue to release, some oil can be driven by water, oil and water are small gradually separated into smaller areas.

2) Heterogeneous model

By initial state, the reservoir in hydrophilic formation model, the irreducible water distribution and the shape of the main: The first one is distributed in the form of water film on the surface of rock particles, and its shape details and thickness of the surface of the rock particles are different from the roughness of the surface; Second is distributed in the small pore and throat; Third are distributed in the pores of small pores or throat traps; Fourth is distributed in a different depth of the "dead end" Lane. It can be seen that the distribution of bound water is connected with the water film on the surface of the large pore rock particles saturated by oil.

\subsection{Local Description of the Experimental Procedure}

When the displacement medium (water) into the reservoir pore system under the action of pressure differential, firstly mixed with bound water, due to the large oil-water viscosity difference, enter into the water of reservoir pore system. On the one hand, flowing through the small pore space that was occupied by the bound water and firstly enter the smaller pores, and then expanded to the larger pores; On the other hand, along with water film of the larger pore wall of the oil saturation wedge, with the increase of the entering water, drainage frontal advancing gradually and the drainaging further intensified. Once the waterline breakthrough, drainage water amount will continue to increase. As shown in Figure 6.

Water flow is not uniform, there is a corresponding flow channel. Thus, we can infer that in the gravity drainage assisted steam flooding process, the condensed water around the steam cavity have corresponding amount channel in the drainage process, rather than uniform drainage. 


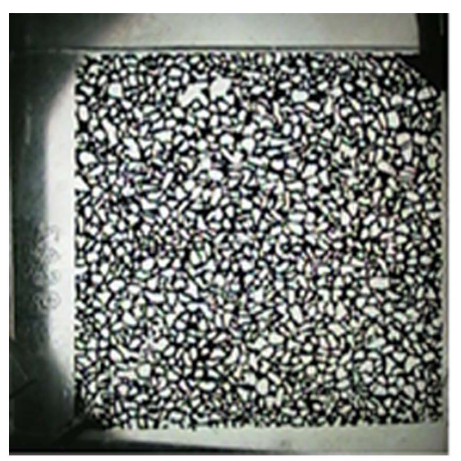

(a) Original saturated core

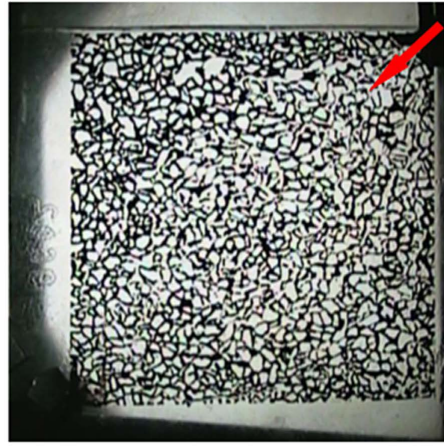

(c) Experimental mediu

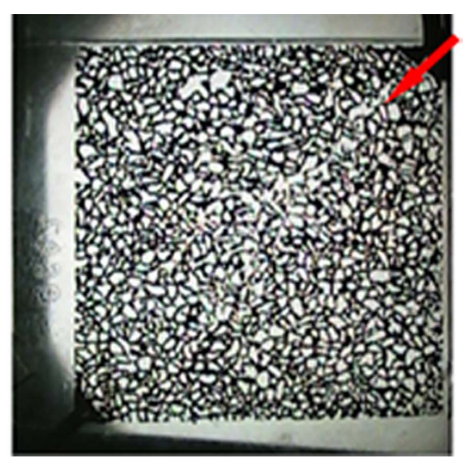

(b) initial experiment

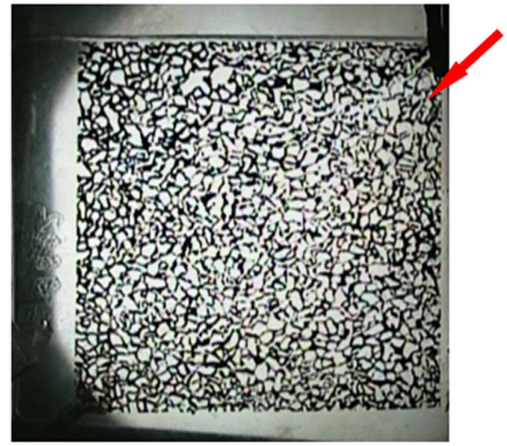

(d) End of experiment stage

Figure 4. The description of homogeneous model drainage process in each period.

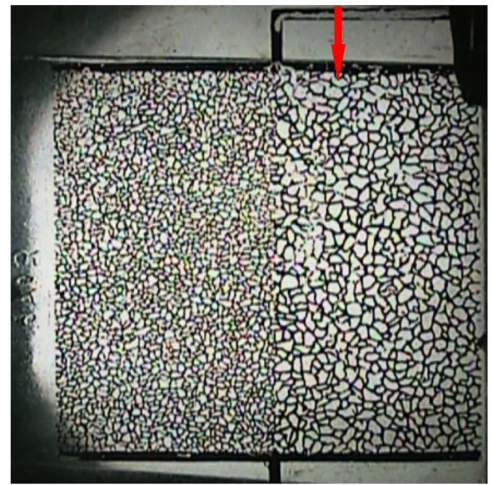

(a) Original saturated core

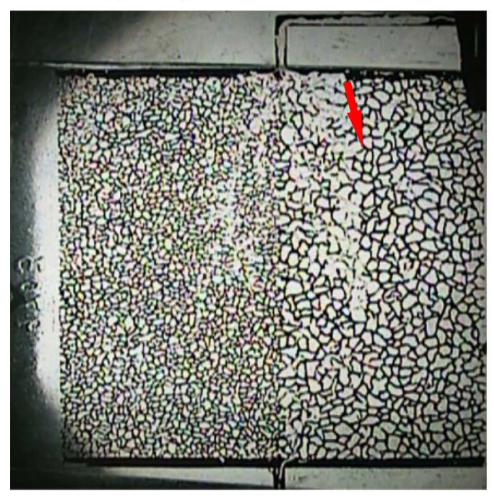

(c) Experimental medium

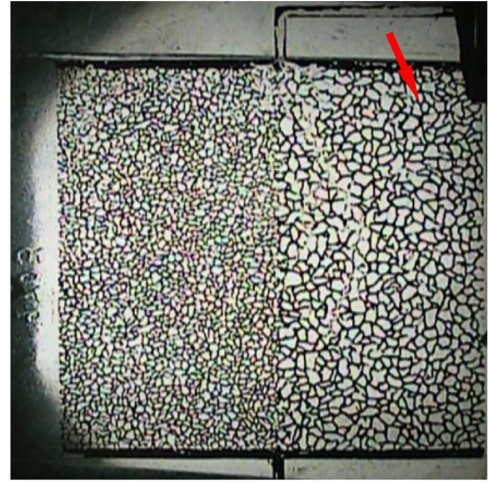

(b) initial experiment

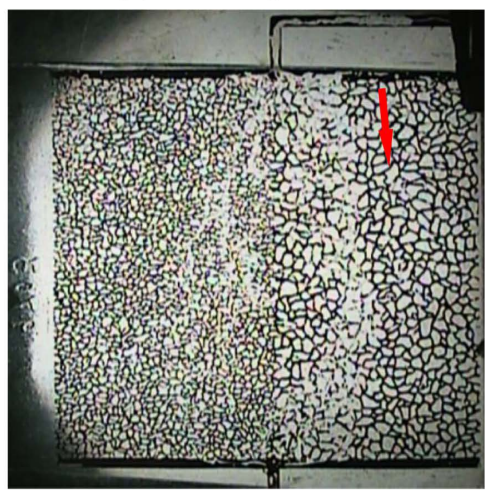

(d) End of experiment stage

Figure 5. The description of heterogeneous model drainage process in each period. 


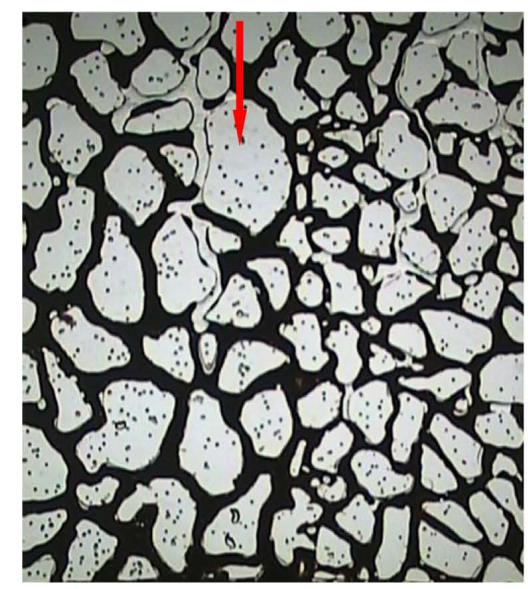

(a)

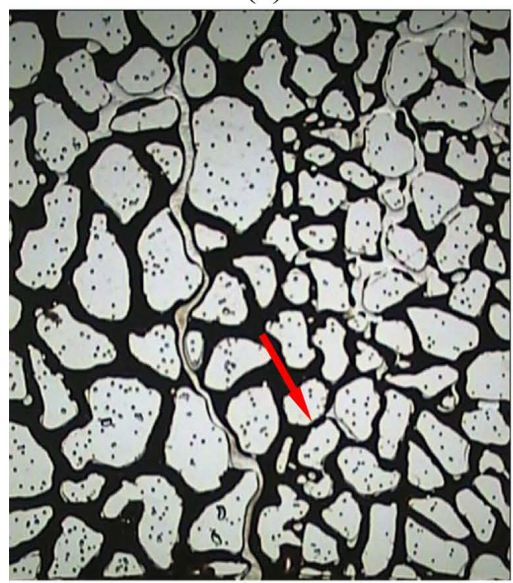

(d)

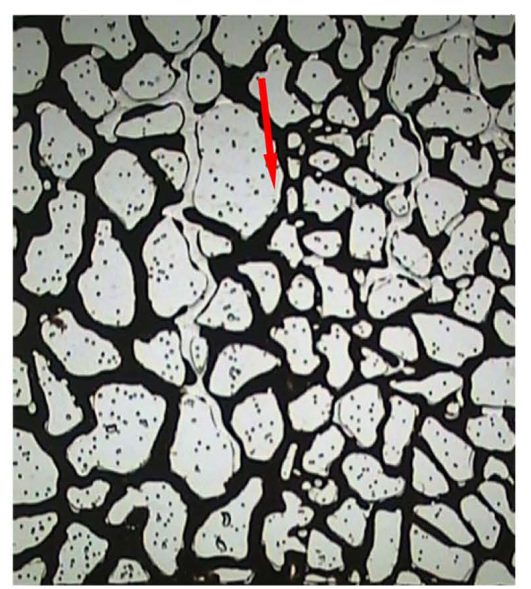

(b)

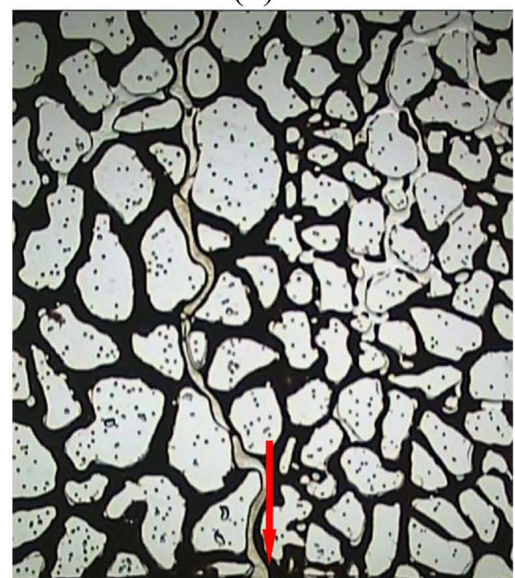

(e)

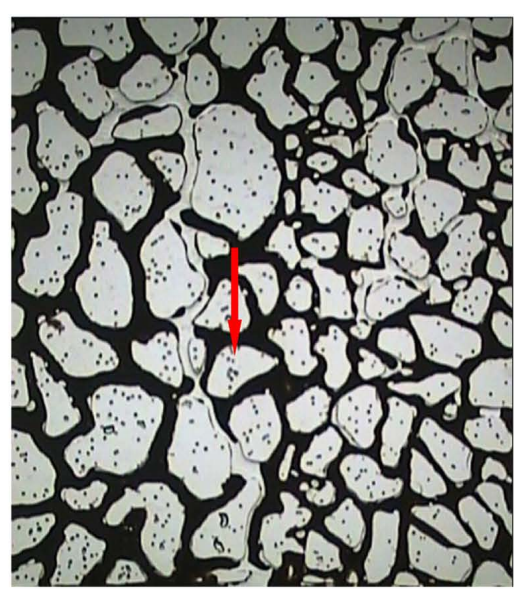

(c)

Figure 6. The local description of heavy oil gravity drainage process.

\subsection{The Sensitivity Analysis of Differential Pressure to Drainage Flow}

1) Homogeneous model

The instantaneous drainage water variation is shown in Figure 7.

Under the different differential pressure is going to have connected and breakthrough state. With the increase of differential pressure, the breakthrough point is advanced. In the early stage of the breakthrough point, almost instantaneous drainage amount phase of different pressure differentials, late in the breakthrough point, the gap is obvious.

2) Heterogeneous model

The instantaneous drainage water variation is shown in Figure 8.

Drainage channels in the heterogeneous model after breakthrough, differential pressure of instantaneous is more sensitive to the influence of water discharge, The increase of instantaneous water discharge caused by the pressure difference of the unit is increased with time.

\section{Conclusion}

The condensed water is not uniform drainage in gravity in the process of water, but along with the drainage channel. In the drainage connection stage, differential pressure has little influence on the instantaneous discharge of water. After the breakthrough, with the increasing of differential pressure, instantaneous drainage water is obvious increase. When the differential pressure is constant, the change of initial instantaneous drainage water is small. After the breakthrough, the instantaneous water discharge increased with time and gradually stabilized. The time of the homogeneous core drain channel formation is early than heterogeneity core. After the 


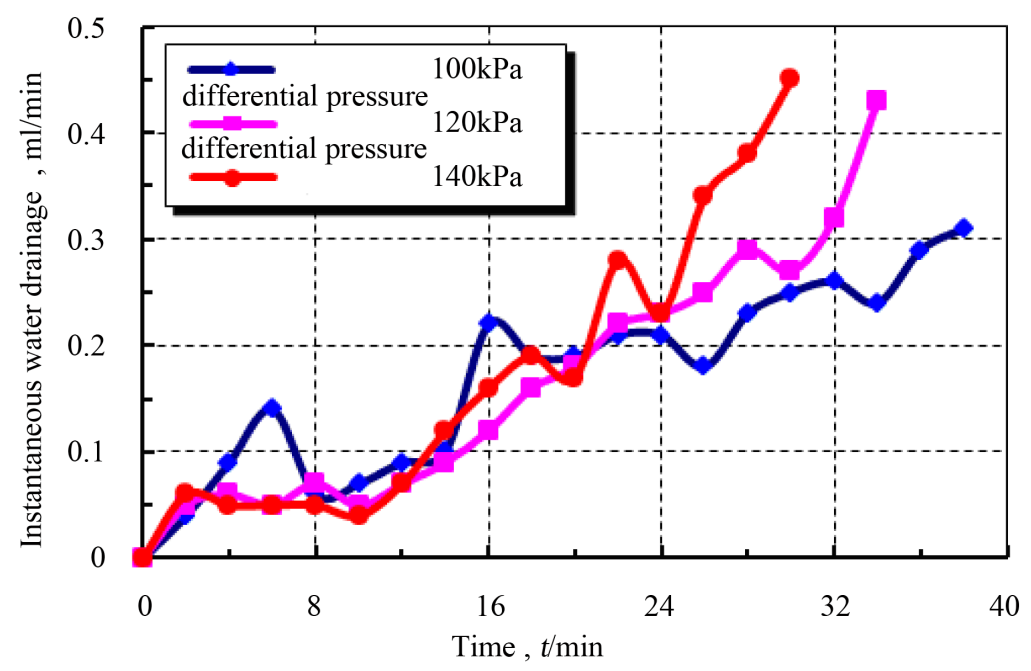

Figure 7. Instantaneous drainage variation curve of homogeneous model.

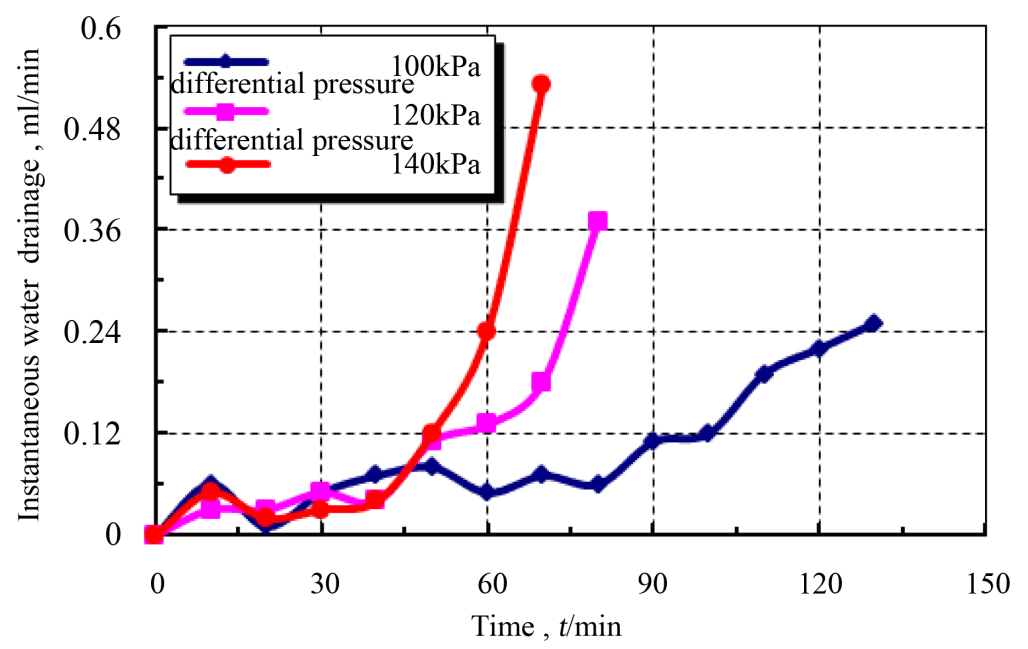

Figure 8. Instantaneous drainage variation curve of heterogeneous model.

formation of drainage channel, the pressure difference of heterogeneous core of instantaneous water impact is greater than the impact on the homogeneous core of instantaneous water.

\section{References}

[1] Liu, D.J., Wang, B., Li, M., et al. (2011) Active Water Flooding Experiment of Micrscopic Visualization. Petroleum Geology and Development of Daqing, 30, 144-147.

[2] Liu, R., Pu, W.F., Peng, Q., et al. (2013) The Effect of Wettability of Porous Media on Micro Macro Efficiency of Polymer Flooding. Oilfield Chemistry, 30, 207-211.

[3] Liu, J.M., Li, Y., Bi, Y.P., et al. (2000) Study on Micro Distribution Characteristics of Remaining Oil in Reservoir by Microscopic Simulation Experiment of Displacement. China Offshore Oil and Gas (Geology), 14, 51-54.

[4] Chen, T. (2007) Micro Water Flooding Experiment of Heterogeneous Reservoir Model. Oil-Gas Geology and Recovery Efficiency, 14, 72-75.

[5] Xia, H.F., Huang, Q.J., Ma, W.G., et al. (2010) Study on the Effect of Non Alkali Two Element Composite System on the Residual Oil after Polymer Flooding in Daqing Oilfield. Oilfield Chemistry, 27, 162-165.

[6] Jia, Z.W., Yang, Q.Y., Lan, Y.B., et al. (2002) Experimental Study on Micro Physical Simulation of Water Drive Oil. Petroleum Geology and Development of Daqing, 21, 46-49. 